\title{
Inducing false lumen thrombosis for retrograde type A aortic dissection
}

Rongjie Zhang, MD, Jian Zhou, MD, Jiaxuan Feng, MD, Zhiqing Zhao, MD, Junjun Liu, MD, Zhenjiang Li, MD, Rui Feng, MD, and Zaiping Jing, MD, PhD

\section{ABSTRACT}

Background: How to choose the proximal landing zone in endovascular interventions for a patient with a patent retrograde false lumen in dissection of the ascending aorta and aortic arch remains unclear. This study sought to report the safety and efficiency of inducing thrombosis of the retrograde false lumen to enhance a proximal landing zone to treat retrograde type A aortic dissection.

Methods: This study included 9 patients with retrograde type A aortic dissection treated with a 2-stage operation strategy between January 2015 and January 2016. Coil and Onyx glue embolization was performed to create a thrombogenic environment in the retrograde false lumen of the ascending aorta and aortic arch as the first-stage operation, followed by thoracic endovascular aortic repair (TEVAR) with the chimney technique as the second-stage operation.

Results: Complete thrombosis in a retrograde false lumen of the ascending aorta and aortic arch was found in all 9 patients before the second-stage operation was performed. The rate of successful TEVAR was $100 \%$, with the single-chimney technique performed in 6 patients to reconstruct the left subclavian artery and the double-chimney technique performed in 3 patients to reconstruct the left common carotid artery. No morbidities, no stent graft-induced new dissection, or deaths occurred in the hospital and during the 12-month follow-up period. Positive morphological remodeling was evidenced in all cases.

Conclusions: The short-term outcomes of the patients in this study were satisfactory. Inducing thrombosis of the retrograde false lumen to enhance the proximal landing zone for TEVAR may be a safe and effective approach to treating retrograde type A aortic dissection. (J Thorac Cardiovasc Surg 2017;153:57-65)

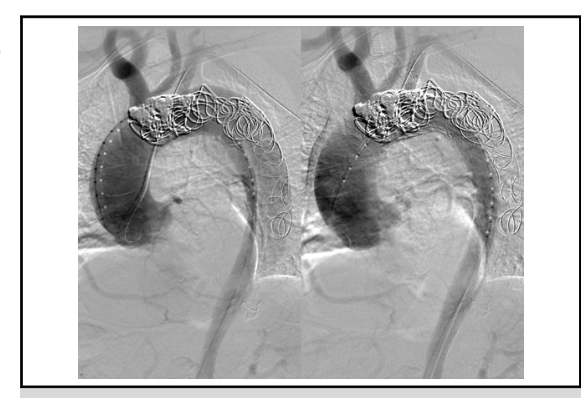

Patient treated by TEVAR after retrograde false lumen inducing thrombosis of the retrograde false lumen.

Central Message

A patent retrograde false lumen can be thrombosed as a stable landing zone for thoracic endovascular aortic repair.

\section{Perspective}

Retrograde type A aortic dissection differs from type $\mathrm{A}$ and type $\mathrm{B}$ aortic dissections. This study evaluated a new treatment strategy for retrograde type A aortic dissection involving coils and Onyx glue to induce thrombosis in the retrograde false lumen, which could provide a proximal landing zone for the second-stage procedure involving thoracic endovascular aortic repair.

See Editorial Commentary page 66.
Retrograde type A aortic dissection (RTAD) is a specific type of Stanford type A aortic dissection (TAAD), defined as aortic dissection with a proximal entry tear in the

\footnotetext{
From the Department of Vascular Surgery, Changhai Hospital, Second Military Medical University, Shanghai, China.

This study was supported by the National Natural Science Foundations of China (Grants 81330034, 81270386, and 81500369), Scientific Research Project of Shanghai Science and Technology Committee (14411963900), and the Shanghai Medical Talents Training Plan (Grant XYQ2013087).

R.Z., J.Z., and J.F. contributed equally to this work and share first authorship.

Received for publication June 27, 2016; revisions received Aug 16, 2016; accepted for publication Sept 2, 2016; available ahead of print Oct 14, 2016.

Address for reprints: Zaiping Jing, MD, PhD, or Rui Feng, MD, Department of Vascular Surgery, Changhai Hospital, 168 Changhai Rd, Shanghai 200433, China (E-mail: xueguanky@163.com or fengrui1588@qq.com).

$0022-5223 / \$ 36.00$

Copyright (C) 2016 by The American Association for Thoracic Surgery http://dx.doi.org/10.1016/j.jtcvs.2016.09.022
}

descending aorta and the false lumen involving the ascending aorta. RTADs constitute an estimated $4 \%$ to $20 \%$ of TAADs. ${ }^{1-4}$ Up to now, treatment of most TAADs has required open surgery with ascending aorta replacement and aortic arch reconstruction. Despite recent advances in surgical techniques and anesthesiology, the perioperative morality of open surgery for TAAD remains high, at $10 \%$ to $35 \%$. $^{5-7}$

Scanning this QR code will take you to a video for the article.

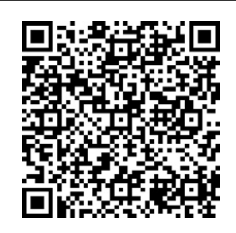




\section{Abbreviations and Acronyms \\ $\mathrm{AD}=$ aortic dissection \\ CTA = computed tomography angiography \\ RTAD = retrograde type A aortic dissection \\ TAAD $=$ type A aortic dissection \\ TBAD $=$ type $\mathrm{B}$ aortic dissection \\ TEVAR $=$ thoracic endovascular aortic repair}

The entry tear of an RTAD is located in the descending aorta, as in Stanford type B aortic dissection (TBAD), but is more difficult to treat with endovascular methods because the proximal landing zone is difficult to define, especially when the false lumen in the ascending aorta and aortic arch is patent. In these circumstances, open surgery with ascending aorta replacement and aortic arch reconstruction may be the sole option.

If the patent retrograde false lumen in the ascending aorta and aortic arch can be transformed into a stable proximal landing zone, then the stent graft can be landed similarly to the procedure for thoracic endovascular aortic repair (TEVAR) for a TBAD. Based on our experience with endovascular repair of aortic dissections in the ascending aorta and aortic arch, ${ }^{8-10}$ we propose a 2-stage strategy aimed at enhancing the safety of TEVAR for an RTAD with a patent retrograde false lumen. The key component of this strategy is the deployment of coils along with Onyx glue to induce thrombosis in the retrograde false lumen, which can then serve as a proximal landing zone in the second-stage procedure. The present study was conducted to evaluate the feasibility of this 2stage strategy for treating retrograde type A aortic dissection.

\section{METHODS \\ Patients}

Between January 2015 and January 2016, patients with an RTAD verified by computed tomography angiography (CTA) were treated in our center. Criteria for inclusion in the present study were (1) RTAD not been treated by open surgery or endovascular repair; (2) patent retrograde false lumen, confirmed by CTA; and (3) minimal false lumen diameter $<20 \mathrm{~mm}$. Exclusion criteria were (1) complicated aortic dissection needing urgent surgery; (2) congenital connective tissue disease, such as Marfan syndrome; (3) any coagulation disorder, such as severe thrombocytopenia; and (4) patient refusal of the 2-stage strategy or inability to comply with the follow-up protocol. The study protocol was approved by our university's Institutional Review Board and Ethics Committee. All patients enrolled in the study provided informed consent for the procedure.

\section{Preoperative Management and Evaluation}

Strict blood pressure and heart rate control were maintained in all patients, using such drugs as urapidil, diltiazem, and nicardipine infused by minipump though a peripheral vein, with gradual transition to an oral agent (eg, valsartan, nifedipine, metoprolol). Analgesic and sedative drugs were given whenever necessary. Three-dimensional reconstruction of the aorta was done by CTA, and anatomic parameters (including diameters at different levels and volumes in true and false lumens) were measured using an Aquarius Workstation (TeraRecon, Foster City, Calif). The status of the false lumen in the ascending aorta and aortic arch was assessed using delayed phase imaging.
Deploying coils in a retrograde false lumen might be not feasible when the false lumen in the thoracic aorta is too large to hold the coils in position (minimal diameter $>20 \mathrm{~mm}$, given the maximum coil diameter of $20 \mathrm{~mm}$ ). For this reason, the anatomic features of the aortic arch, the retrograde extension and morphological characteristics of the false lumen, and the location of proximal and distal tears must be carefully evaluated.

Acute aortic dissection was defined as aortic dissection occurring within 14 days of onset of symptoms; chronic aortic dissection, as dissection occurring later. ${ }^{11}$ The first-stage operation of this 2-stage strategy is performed in the chronic phase because the endovascular intervention requires a mature flap and a stable patient.

\section{First-Stage Operation Procedure}

All of the first-stage operations were performed under local anesthesia. The choice of the left or right femoral artery as the access artery was based on the anatomic characteristics of the aortic dissection. Once aortography confirmed the location and branch artery involvement of the aortic dissection, a blood pressure transducer was connected to a $5 \mathrm{~F}$ pigtail catheter to measure blood pressure at different levels of the true lumen from the iliac artery to the ascending aorta.

Next, a 0.035 -inch guide wire was advanced to the false lumen of the ascending aorta through a distal tear in the descending or abdominal aorta. On angiography of the false lumen, blood pressure in the false lumen was measured at the same levels as for the true lumen. Detachable coils (Interlock-35; Boston Scientific, Watertown, Mass) were deployed into the retrograde false lumen.

Two conditions can occur during the coil deploying process: the coils can remain in the retrograde false lumen along the ascending aorta and aortic arch, or can drift to the descending aorta. The narrow part or rotation of the false lumen along the descending aorta could hold the coils. From this "base," subsequent coils could be gradually deployed into the retrograde false lumen.

To make the thrombus fill the entire retrograde false lumen, a microcatheter (Echelon-10; ev3 Neuroavascular, Irvine, Calif) was introduced, through which Onyx glue (ev3 Neurovascular) was injected into the retrograde false lumen. The volume of Onyx glue injected was estimated according to anatomic parameters. During the injection procedure, glue injection was stopped based on the pulsing movement of the deployed coils and angiography in the false lumen. After injection, blood pressure in the false lumen was remeasured through the $5 \mathrm{~F}$ pigtail catheter.

\section{Management and Evaluation Between Operations}

Blood pressure and heart rate were strictly controlled. Analgesic and sedative drugs were administered when necessary. Neither anticoagulants nor coagulants were administered. Approximately 2 weeks after firststage operation, CTA and 3-dimensional reconstruction were examined to confirm the thrombosis in the false lumen. Based on the findings of this examination, a detailed strategy for the second-stage operation was determined, including the diameter and length of the main stent-graft and chimney stents.

\section{Second-Stage Operation Procedure}

All second-stage operations were performed with the patient under general anesthesia. To create a proximal landing zone of at least $20 \mathrm{~mm}$, the anchoring site was planned for zone 1 or zone $2 .{ }^{12}$ Two chimney stents (in the left carotid artery and left subclavian artery) or a single chimney stent (in the left subclavian artery) were used to preserve the arch branch arteries. A final aortography confirmed the patency of the arch branches and the exclusion of the false lumen.

\section{Follow-up}

CTA examination and 3-dimensional reconstruction were performed at 3 months, 6 months, and then annually after the second-stage operation. Video 1 shows the entire process of this 2-stage strategy for treating a 


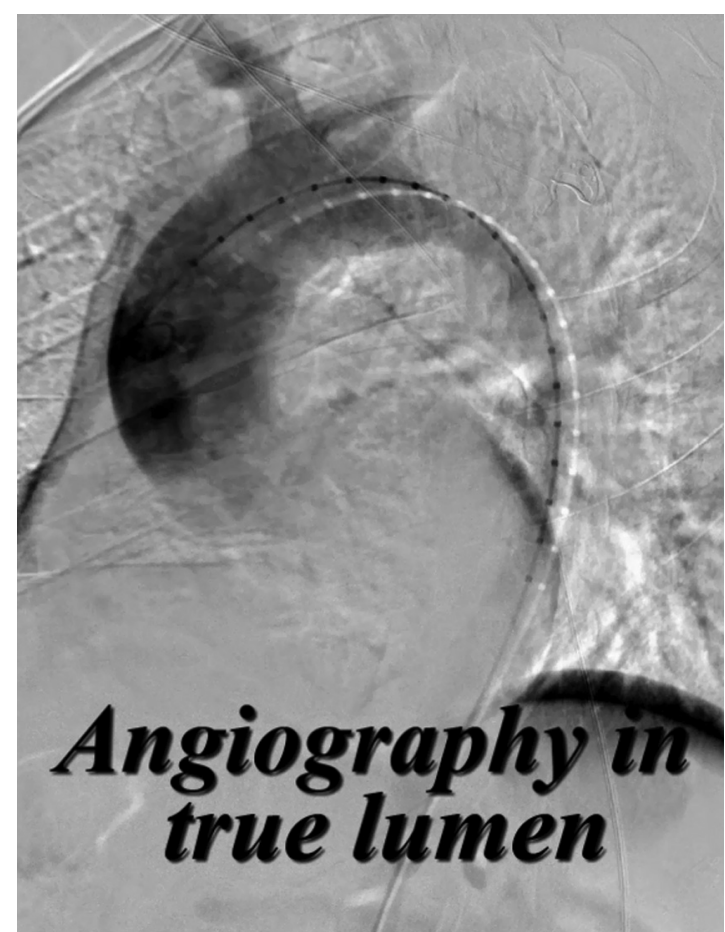

VIDEO 1. Computed tomography angiography showing 3D reconstructions (preoperative, after first-stage operation, and after the second-stage operation), and during the first- and second-stage operations showing the 2-stage approach to treating retrograde type A aortic dissection. Video available at: http://www.jtcvsonline.org/article/S0022-5223(16)31140-0/ addons.

patient with RTAD. Thrombosis of the false lumen, morphology of the true lumen, and patency of the chimney stents were evaluated. The diameters of both the true lumen and the total aorta at the level of the maximum dilation of the thoracic descending aorta were recorded. Emergent CTA examinations were obtained in a patient demonstrating signs or symptoms of adverse events. All evaluations were done by vascular surgeons.

\section{Statistical Analysis}

Continuous variables were summarized as mean $\pm \mathrm{SD}$, and skewed variables were summarized as median and range. Data were analyzed using SPSS for Windows (IBM, Armonk, NY). Paired $t$ test analyses were performed for true lumen blood pressure and false lumen blood pressure before thrombosis, and also for false lumen blood pressure before and after thrombosis. In addition, paired $t$ test analyses were performed for the diameters of the total aorta and the true lumen at the level of maximum dilation of the thoracic descending aorta before the interventions and again at 3 months after the second-stage operation.

\section{RESULTS}

Fifteen patients were diagnosed with RTAD in our hospital between January 2015 and January 2016. One patient sustained sudden death in emergency room, and 5 patients were excluded, including 2 patients with the need for urgent open surgery, 1 patient with Marfan syndrome, 1 patient with a minimal false lumen diameter of $>20 \mathrm{~mm}$, and 1 patient who did not accept the 2-stage strategy and other medical management. The 2 patients who required urgent
TABLE 1. Patient characteristics and clinical outcomes $(n=9)$

\begin{tabular}{lc}
\hline \multicolumn{1}{c}{ Characteristic } & Value \\
\hline Male sex, n (\%) & $8(88.9)$ \\
Age, yr, mean \pm SD & $52.0 \pm 9.4$ \\
Chest pain, n $(\%)$ & $8(88.9)$ \\
Ischemic symptoms, n & 0 \\
Hypertension, n $(\%)$ & $5(55.6)$ \\
Diabetes mellitus, n (\%) & $3(33.3)$ \\
Smoking, n (\%) & $7(77.8)$ \\
Time from O to F, d, mean \pm SD (range)* & $15.8 \pm 1.9(14-20)$ \\
Time from F to S, d, mean \pm SD (range) & $15.3 \pm 2.8(11-19)$ \\
Duration of hospitalization, d & $28.3 \pm 7.0$ \\
In-hospital complications, $\mathrm{n}$ & 0 \\
Follow-up duration, mo, median (range) & $12(6-18)$ \\
Follow-up complications, $\mathrm{n}$ & 0 \\
\hline
\end{tabular}

$O$, Onset; $F$, first-stage operation; $S$, second-stage operation. *One asymptomatic patient was not included in this calculation because his actual onset time could not be determined and his case was presumed to be chronic.

open surgery had carotid artery involvement, verified by CTA, and had sustained a transient ischemic attack. After exclusions, a total of 9 patients who underwent the 2-stage operation were included in the present analysis.

Demographic data for these 9 patients are presented in Table 1. The mean patient age was $52.0 \pm 9.4$ years (range, 41 to 67 years). All 9 patients had uncomplicated dissection (without malperfusion syndrome or signs of rupture, such as pleural effusion, refractory pain, or rapid aortic expansion) at onset or during the hospital stay. The mean interval from onset to the first-stage operation was $15.8 \pm 1.9$ days in 8 patients (range, 14 to 20 days); 1 asymptomatic patient is not included in this calculation because his actual time of onset could not be determined, and thus his dissection was deemed chronic. The mean interval from the first-stage operation to the second-stage operation was $15.3 \pm 2.8$ days (range, 11 to 19 days). No patient experienced any complications during hospitalization or follow-up.

Details of the 2 operations are presented in Table 2. In the first-stage operation, between 6 and 13 (median, 11) coils

TABLE 2. Characteristics of the 2 operations

\begin{tabular}{lc}
\multicolumn{1}{c}{ Characteristic } & Value \\
\hline First-stage operation & \\
$\quad$ Number of coils, median (range) & $11(7-13)$ \\
$\quad$ Volume of onyx glue, $\mathrm{mL}$ & $3.94 \pm 1.18$ \\
$\quad$ Procedure time, min & $98.3 \pm 19.0$ \\
Second-stage operation & \\
$\quad$ Retrograde false lumen thrombosis, $\mathrm{n}(\%)$ & $9(100)$ \\
Single-chimney technique, $\mathrm{n}(\%)$ & $6(66.7)$ \\
Double-chimney technique, $\mathrm{n}(\%)$ & $3(33.3)$ \\
Aortic diameter (proximal to the tear), mm, mean $\pm \mathrm{SD}$ & $28.4 \pm 3.4$ \\
Main stent diameter, mm, mean $\pm \mathrm{SD}$ & $35.6 \pm 4.5$ \\
Main stent oversizing, $\%$, mean $\pm \mathrm{SD} *$ & $16.4 \pm 2.3$ \\
Procedure time, min, mean $\pm \mathrm{SD}$ & $102.2 \pm 44.2$ \\
\hline *Oversizing was defined as (main stent diameter - aortic diameter)/aortic diameter.
\end{tabular}




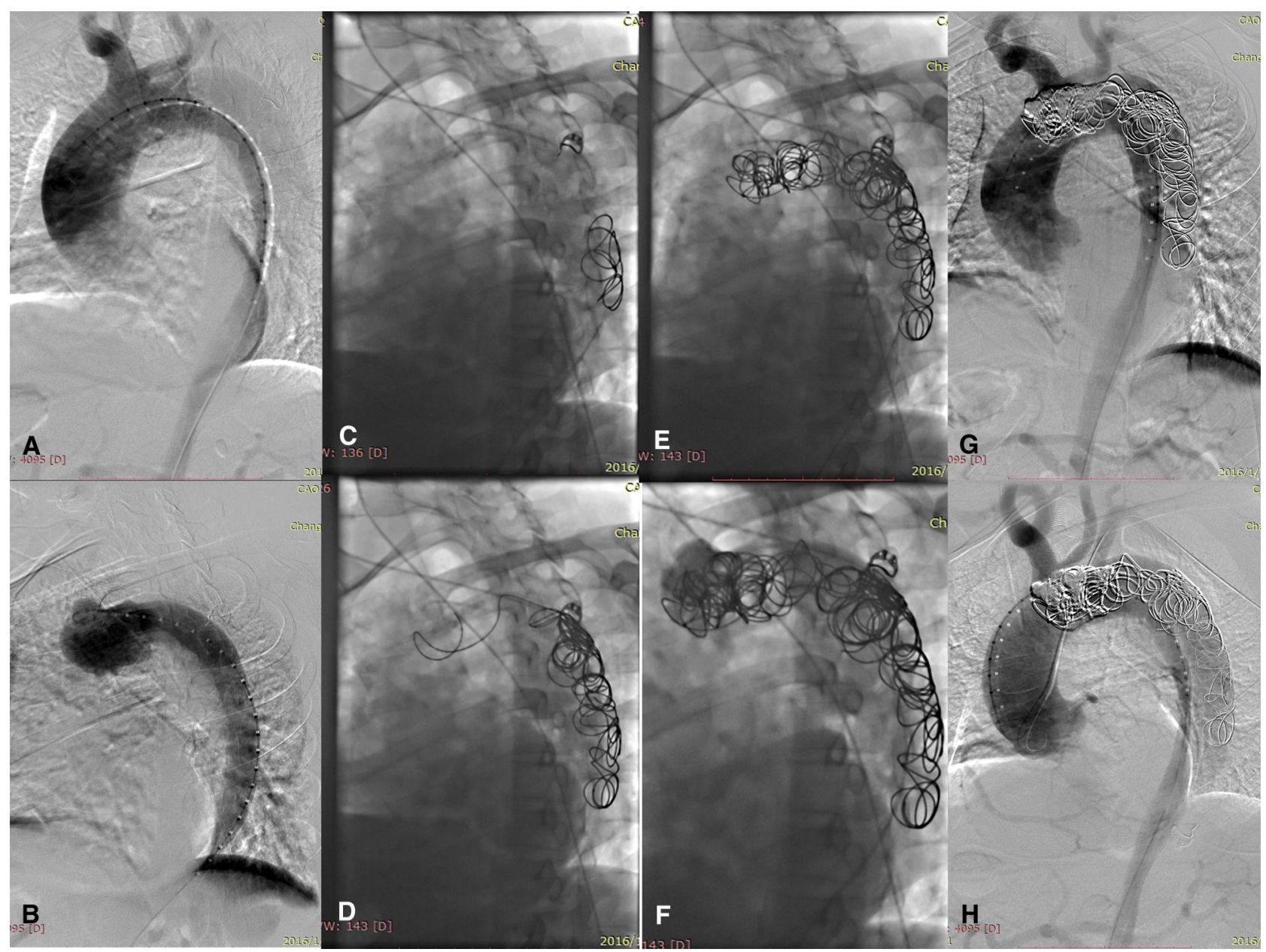

FIGURE 1. A, Computed tomography aortography in the true lumen. B, Aortography in the false lumen. C, The first coil released, showing coil drift to the middle part of the thoracic aorta. D, The sixth coil released, with the coil zone extended to the aortic arch. E, The 13th coil released, with the coil zone extended to the ascending aorta. F, Onyx glue injection to enhance firmness. G, Aortography after the first operation, showing a satisfactory retrograde false lumen embolism. H, Aortography before the second operation, at 14 days after embolism.

were used in each patient. Coils drifted to the middle thoracic aorta at the beginning of embolization in 4 patients and to the proximal thoracic aorta in 3 patients, but the embolism area could extend to aortic arch and ascending aorta after coils released one by one (Figure 1). There were 2 cases without drifted coils; the coils stayed in the false lumen along the ascending aorta. All patients underwent Onyx glue injection; the mean volume of glue injected was $3.94 \pm 1.18 \mathrm{~mL}$. Blood pressure measurements obtained at different levels of the aorta are shown in Figure 2.

At the second-stage operation, complete false lumen thrombosis in the ascending aorta and aortic arch were found in all 9 patients. A single-chimney technique was performed in 6 patients, and a double-chimney technique was performed in the other 3 patients. The mean oversizing of main stents was $16.4 \% \pm 2.3 \%$. Angiography after stenting demonstrated complete exclusion in 8 patients with no endoleak and in 1 patient with a small type Ia endoleak.
The median duration of follow-up was 12 months (range, 6 to 18 months). No cerebral infarctions, no new dissections, and no chimney stent stenosis or occlusion were recorded during the follow-up period. In the patient with a small type Ia endoleak, the endoleak had disappeared by the 3-month follow-up, and no new endoleaks were detected. No other complications or deaths were recorded during the follow-up period. The mean diameter of the total aorta at the level of maximal dilation of the thoracic descending aorta was $31.0 \pm 3.7 \mathrm{~mm}$ before intervention and $31.6 \pm 3.7 \mathrm{~mm}$ at 3 months after the second-stage operation $(P=.21)$. The corresponding mean diameters of the true lumen were $20.8 \pm 4.3 \mathrm{~mm}$ and $27.4 \pm 4.2 \mathrm{~mm}(P<.01)$. Complete thrombosis of the false lumen of the ascending aorta and aortic arch were noted in all 9 patients (Figure 3). Complete thrombosis of the false lumen of the thoracic descending aorta was seen in 8 patients $(88.9 \%)$, and partial thrombosis was noted in 1 patient $(11.1 \%)$. 

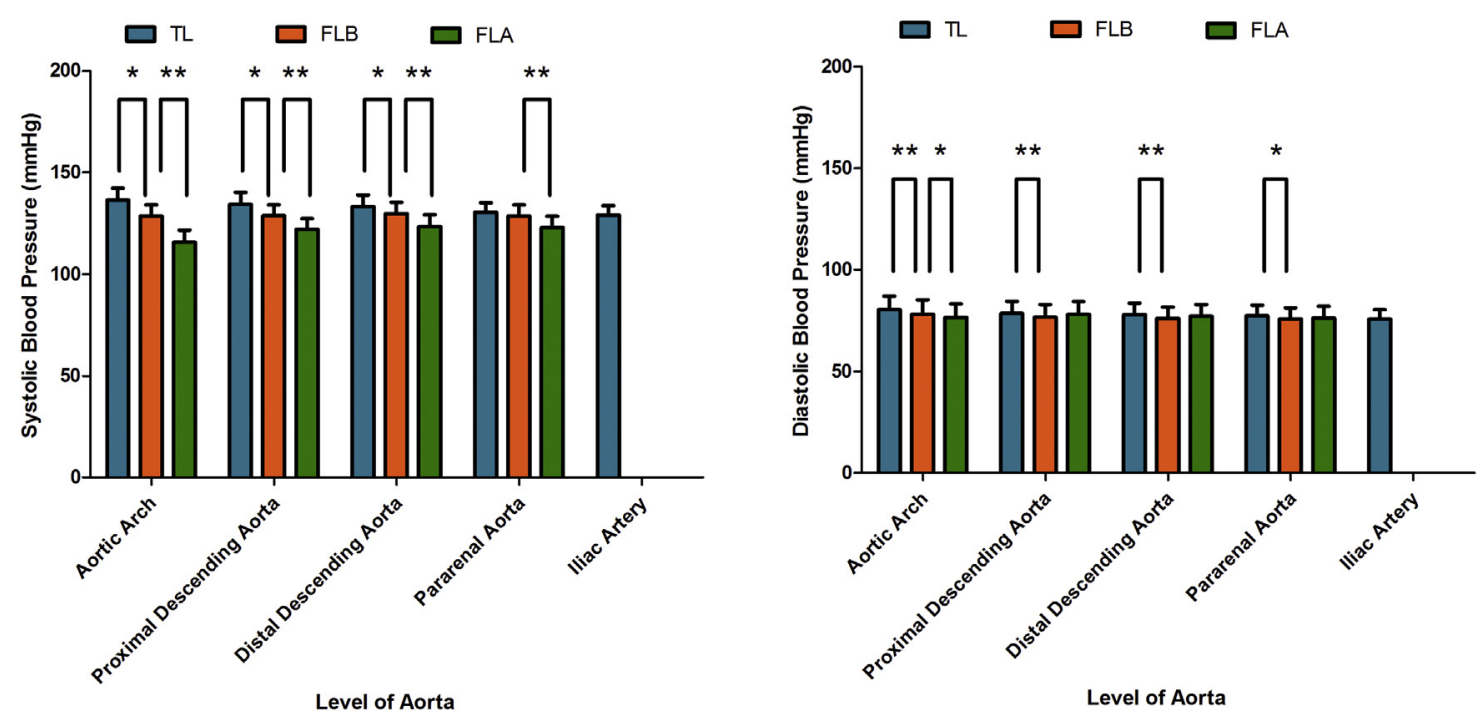

FIGURE 2. Blood pressure at different levels of the aorta in the first-stage operation. $T L$, True lumen; $F L B$, false lumen before embolization; $F L A$, false lumen after embolization. ${ }^{*} P<.05 ; * * P<.001$

Figure 4 illustrates the 2-stage strategy for managing RTAD. Of the 2 patients who underwent urgent open surgery in our hospital, 1 experienced sudden death around 6 months after surgery, and the other was alive at the 15-month follow-up.

\section{DISCUSSION}

Since it was first applied in aortic dissection by Dake and colleagues in $1994^{13}$ and then first systematically summarized as TEVAR by Nienaber and colleagues $1999,{ }^{14}$ TEVAR has been applied to a multitude of aortic diseases, ${ }^{15}$ and is now considered an optimal treatment approach for TBAD. RTAD is a specific type of TAAD; although the ascending aorta is affected in both types, hemodynamics, the progress of dissection, and prognosis differ. ${ }^{16}$ Even though in RTAD the entry tear is located in the descending aorta, RTAD is more difficult to treat endovascularly than TBAD, owing to the lack of a proximal unaffected aorta to serve as a stent-graft landing zone. Thus, RTAD requires a unique therapeutic regimen.

As for open surgery, Segesser and coworkers ${ }^{17}$ have suggested that RTAD should be managed based on the site of the predominant lesion, and that endovascular treatment for RTAD should depend on its predominant lesion as well as on involved regions. Kaji and colleagues ${ }^{18}$ have suggested that an RTAD with a nonthrombosed false lumen in the ascending aorta necessitates open surgery. Kim and coworkers $^{16}$ have recommended that a patient with a thrombosis in the ascending aortic false lumen but without a severely dilated ascending aorta (ie, $<5.5 \mathrm{~cm}$ ) be considered for medical management combined with timely interventions. ${ }^{16}$ Although this approach was suggested for open surgery, it also may be applicable for endovascular therapy, which is simpler and much less invasive than open surgery, and may help reduce operative and postoperative mortality and morbidity.

Kato and colleagues ${ }^{19}$ reported the successful use of an endovascular technique with stent grafts to treat RTAD in 10 patients, with complete thrombosis of the false lumen of the ascending and descending aorta by 3 months after stent grafting. They suggested that the endovascular technique is a safe and effective method, but should be used only in carefully selected patients, with stent grafts deployed only distal to the left subclavian artery. In our series of patients, however, there was an insufficient proximal landing zone distal to the left subclavian artery, and we considered the patent retrograde false lumen along the ascending aorta and aortic arch too frail to serve as a proximal landing zone.

Previous studies have identified different features of an RTAD with a thrombosed false lumen in the ascending aorta compared with an antegrade TAAD. ${ }^{16,18}$ An already thrombosed false lumen in the ascending aorta can be treated in the same manner as a TBAD.$^{17}$ Because false lumen thrombosis is an important factor to consider when determining the treatment strategy for RTAD, we sought to thrombose the retrograde false lumen so as to create a proximal landing zone for TEVAR. In this way, an RTAD with a patent retrograde false lumen could be treated in the same manner as a TBAD.

Coil embolization has been widely used in the endovascular treatment of many types of vascular diseases and abnormalities. Coils also have been used in the treatment of aortic dissection. Almost of the reported cases were involved remedial or assisted therapeutic methods to deal with a patent false lumen after TEVAR or surgical 


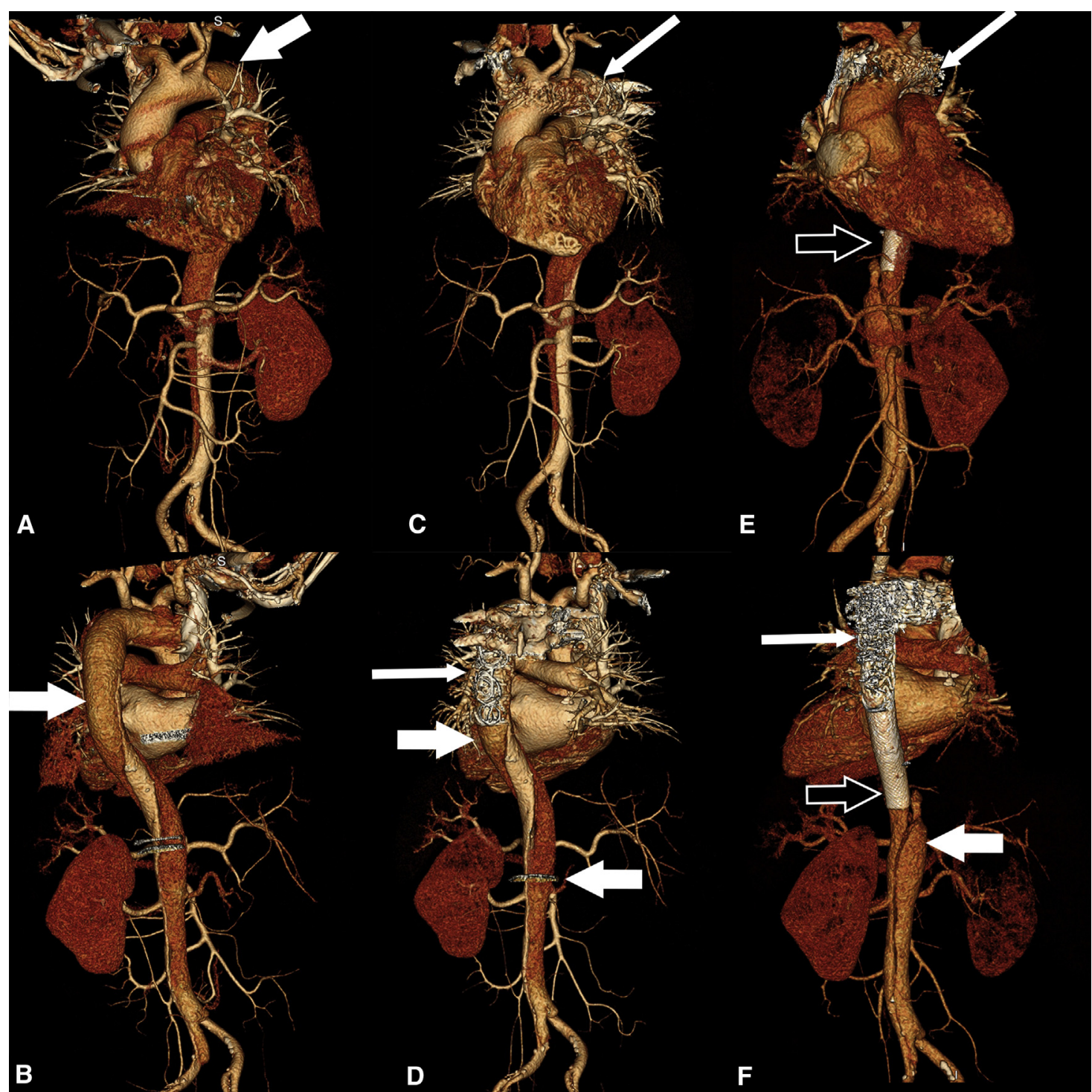

FIGURE 3. A and B, Computed tomography angiography (CTA) reconstruction before the first-stage operation, showing the false lumen (short white arrows). C and D, CTA reconstruction after the first-stage operation, showing coils in the thrombus area (long white arrows). E and F, CTA reconstruction at 3 months after the second-stage operation, showing the stent graft (black arrows).

intervention. ${ }^{20-22}$ Katayama and coworkers ${ }^{23}$ used coils to cover the distal reentry tear before standard TEVAR in a patient with chronic TBAD. Outcomes of coil treatment reported to date have been satisfactory in all cases.

A permanent liquid embolic agent, Onyx glue has been widely used in the treatment of many vascular disorders. Previous studies have suggested that the use of coils along with Onyx glue is a safe and efficient approach to providing a durable thrombogenic environment. ${ }^{24-27}$ The use of coils and Onyx glue can mitigate false lumen flow, extending the zone of thrombosis to create a sufficient proximal landing zone for TEVAR to treat RTAD. Much like steelreinforced concrete provides a solid building material, coil-reinforced Onyx glue can provide a firm thrombosis zone.
In our series of patients with RTAD, the initial tear was located at the descending aorta, affecting the aortic arch to the ascending aorta. Coils combined with Onyx glue were used in the first-stage operation to create the retrograde false lumen thrombosis. We assumed that this approach would prevent further dissection to the proximal part, especially during the prestenting period, and also provide a stable anchoring environment for the stent graft. We noted no retrograde progress of dissection during the second-stage operation, preliminarily confirming the effectiveness of the induced thrombosis in these patients.

In the first-stage operation, coils often drifted to the distal part even when released at the ascending false lumen, owing to the high blood flow velocity in the retrograde false lumen. We speculate that it is very difficult to create a false lumen 

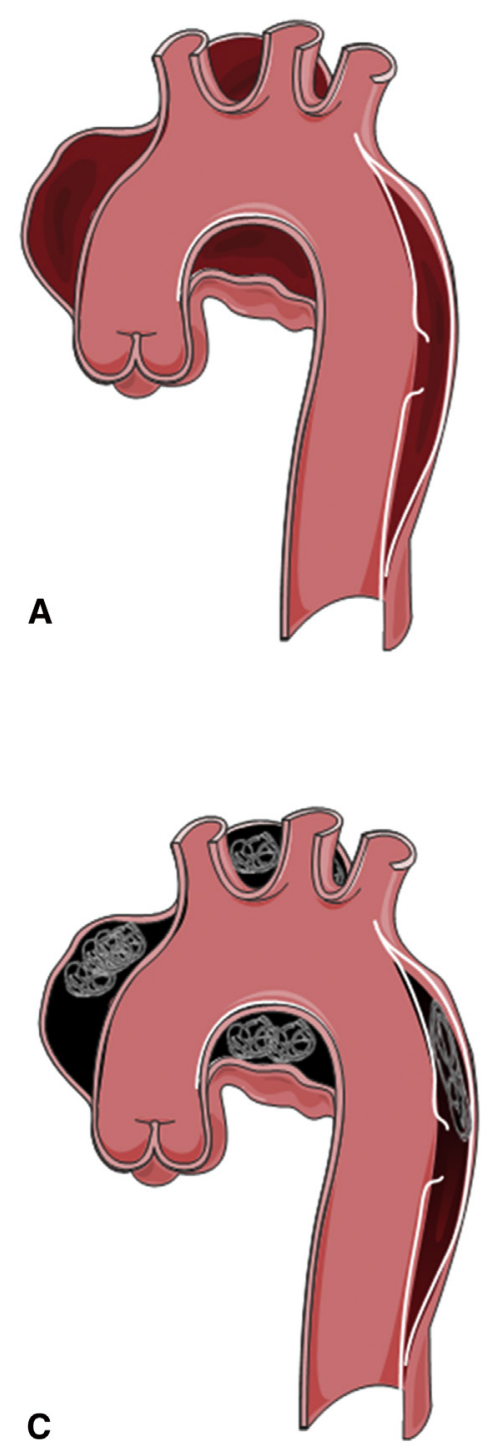
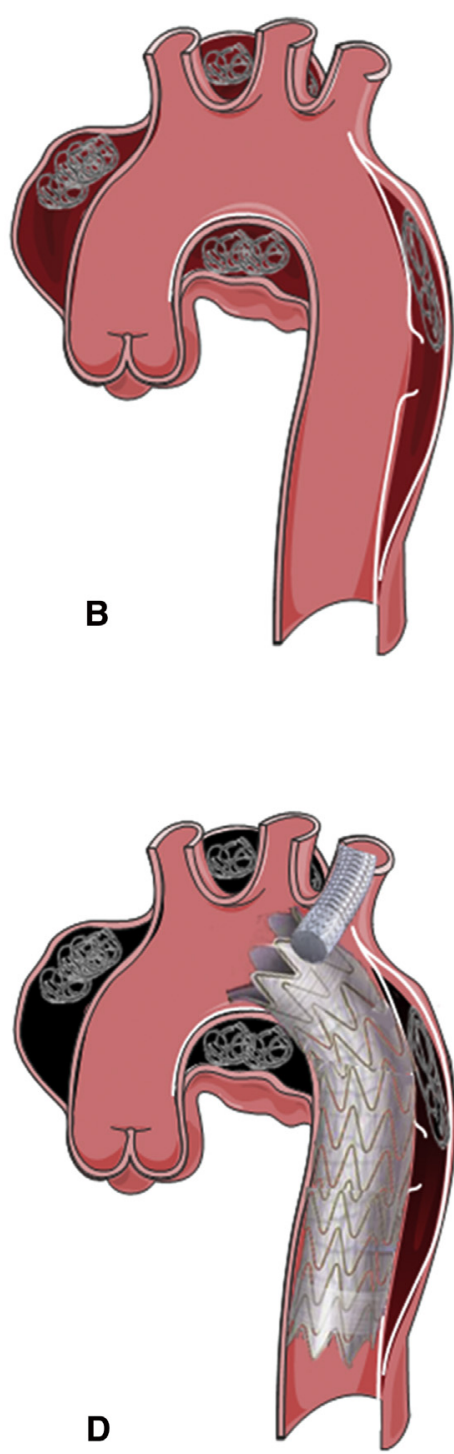

FIGURE 4. Schematic diagram of the 2-stage strategy for managing retrograde type A aortic dissection (RTAD). A, The RTAD. B, Coil thrombosis of the retrograde false lumen. C, Retrograde false lumen thrombosed. D, Thrombosis area as a proximal landing zone for thoracic endovascular aortic repair.

thrombosis solely by conservative medical treatment. A chaotic hemodynamic status and high false lumen blood pressure before embolization may cause further dissection to the proximal part (Figure 2). Systolic blood pressure in the false lumen was decreased at all 4 levels measured after thrombosis, whereas diastolic blood pressure was only decreased at the aortic arch after the coils and Onyx glue were deployed. Hemodynamic status changed on use of the embolic material.

As shown in Figure 1, coils often drifted to the distal part at the start of embolization. Complete false lumen thrombosis in the ascending aorta and aortic arch could not be achieved if insufficient embolism was released; thus, a large number of coils was used, between 7 and 13 (median, 11) in each patient. Effective coagulation and thrombosis could not be achieved if the Onyx glue was injected without coils or before the coils released. Embolus adhesion and coagulation are very important in this process, to avoid drifting; thus, the coils must be of sufficient length and properly fiber-coated. Embolus materials and coagulation performance require further investigation.

Stent-induced new tears or RTADs are rare but serious complications after TEVAR. ${ }^{28-31}$ Stent-induced RTAD can result from manipulation of guidewires and sheaths, as well as the large-bore stent delivery system within the aortic arch during the operation. It has been reported during hospitalization as well as up to 1050 days after the procedure. ${ }^{32}$ The aortic tissue is fragile in patients with aortic dissection, and thus even nondissected portions of the aorta may be vulnerable. ${ }^{29}$ Stent grafts are more likely to cause new dissection in the vulnerable aortic wall. Creation of a man-made thrombus can provide a stable stent graft 
anchorage area and thereby reduce the number of chimney stents required in the aortic arch.

The chimney technique was used in our 9 patients owing to an insufficient anchor area for main stents. Given that the reported incidence of endoleak associated with the chimney technique is as high as $26 \%,{ }^{33}$ main stent oversizing should be aggressive to help avoid endoleak. Overaggressive oversizing of the main stent may cause harm to the aortic wall, however, possibly increasing the risk of RTAD. Nevertheless, because the man-made thrombus area provides a more stable anchor environment, oversizing of the main stent might not pose a dilemma.

\section{Limitations}

Our experience in the 9 patients in the present series indicates that the 2-stage operation was feasible for all planned procedures. Aortic remolding was noted after the 2-stage operation. Limitations of this study are the small sample size $(n=9)$ and short median follow-up (12 months). Further studies with larger sample sizes and longer follow-up are needed. Future studies also should compare the results of medical management, a combination of induced thrombosis and medical management, and the 2-stage procedure for RTAD.

\section{CONCLUSIONS}

In patients with RTAD, a thrombogenic environment in the false lumen of the ascending aorta and aortic arch can be created using coils and Onyx glue, extending the thrombosis zone to provide a platform for anchoring endovascular stents. Creation of this man-made thrombosis of the false lumen in the ascending aorta and aortic arch before TEVAR may be a safe and effective treatment strategy for RTAD. In the present study, short-term patient outcomes were satisfactory; however, long-term outcomes remain to be evaluated.

\section{Conflict of Interest Statement}

Authors have nothing to disclose with regard to commercial support.

\section{References}

1. Lansman SL, Raissi S, Ergin MA, Griepp RB. Urgent operation for acute transverse aortic arch dissection. J Thorac Cardiovasc Surg. 1989;97:334-41.

2. Kazui T, Tamiya Y, Tanaka T, Komatsu S. Extended aortic replacement for acute type A dissection with the tear in the descending aorta. J Thorac Cardiovasc Surg. 1996;112:973-8.

3. Lansman SL, Galla JD, Schor JS, Ergin MA, Griepp RB. Subtypes of acute aortic dissection. J Card Surg. 1994;9:729-33.

4. Daily PO, Trueblood HW, Stinson EB, Wuerflein RD, Shumway NE. Management of acute aortic dissections. Ann Thorac Surg. 1970;10:237-47.

5. Hagan PG, Nienaber CA, Isselbacher EM, Bruckman D, Karavite DJ, Russman PL, et al. The International Registry of Acute Aortic Dissection (IRAD): new insights into an old disease. JAMA. 2000;283:897-903.

6. Kouchoukos NT, Dougenis D. Surgery of the thoracic aorta. N Engl J Med. 1997; 336:1876-88.
7. Lai DT, Robbins RC, Mitchell RS, Moore KA, Oyer PE, Shumway NE, et al. Does profound hypothermic circulatory arrest improve survival in patients with acute type A aortic dissection? Circulation. 2002;106:I218-28.

8. Feng R, Zhao Z, Bao J, Wei X, Wang L, Jing Z. Double-chimney technology for treating secondary type I endoleak after endovascular repair for complicated thoracic aortic dissection. J Vasc Surg. 2011;54:212-5.

9. Lu Q, Feng J, Zhou J, Zhao Z, Bao J, Feng R, et al. Endovascular repair of ascending aortic dissection: a novel treatment option for patients judged unfit for direct surgical repair. J Am Coll Cardiol. 2013;61:1917-24.

10. Lu Q, Feng J, Zhou J, Zhao Z, Li H, Teng Z, et al. Endovascular repair by customized branched stent-graft: a promising treatment for chronic aortic dissection involving the arch branches. J Thorac Cardiovasc Surg. 2015;150: 1631-8.e5.

11. Hirst AE Jr, Johns VJ Jr, Kime SW Jr. Dissecting aneurysm of the aorta: a review of 505 cases. Medicine (Baltimore). 1958;37:217-79.

12. Fillinger MF, Greenberg RK, McKinsey JF, Chaikof EL. Reporting standards for thoracic endovascular aortic repair (TEVAR). J Vasc Surg. 2010;52: 1022-33.e15.

13. Dake MD, Miller DC, Semba CP, Mitchell RS, Walker PJ, Liddell RP. Transluminal placement of endovascular stent-grafts for the treatment of descending thoracic aortic aneurysms. N Engl J Med. 1994;331:1729-34.

14. Nienaber CA, Fattori R, Lund G, Dieckmann C, Wolf W, von Kodolitsch Y, et al. Nonsurgical reconstruction of thoracic aortic dissection by stent-graft placement. N Engl J Med. 1999;340:1539-45.

15. Schaffer JM, Lingala B, Miller DC, Woo YJ, Mitchell RS, Dake MD. Midterm survival after thoracic endovascular aortic repair in more than 10,000 Medicare patients. J Thorac Cardiovasc Surg. 2015;149:808-20.

16. Kim JB, Choo SJ, Kim WK, Kim HJ, Jung SH, Chung CH, et al. Outcomes of acute retrograde type A aortic dissection with an entry tear in descending aorta. Circulation. 2014;130(11 Suppl 1):S39-44.

17. von Segesser LK, Killer I, Ziswiler M, Linka A, Ritter M, Jenni R, et al. Dissection of the descending thoracic aorta extending into the ascending aorta: a therapeutic challenge. J Thorac Cardiovasc Surg. 1994;108:755-61.

18. Kaji S, Akasaka T, Katayama M, Yamamuro A, Yamabe K, Tamita K, et al. Prognosis of retrograde dissection from the descending to the ascending aorta. Circulation. 2003;108(Suppl 1):II300-6.

19. Kato N, Shimono T, Hirano T, Ishida M, Yada I, Takeda K. Transluminal placement of endovascular stent-grafts for the treatment of type A aortic dissection with an entry tear in the descending thoracic aorta. J Vasc Surg. 2001;34:1023-8.

20. Jeganathan R, Kennedy P, MacGowan S. Treatment of a persistent false lumen with aneurysm formation following surgical repair of type a dissection. Cardiovasc Intervent Radiol. 2007;30:491-3.

21. Norberto EM, Gutiérrez VM, Taylor J, Vaquero C. Coil embolization of persistent false lumen after stent graft repair of type B aortic dissection. J Vasc Surg. 2011;54:201-4.

22. Hager E, Moudgill N, Lipman A, Dimuzio P, Lombardi J. Coil-assisted false lumen thrombosis in complicated chronic type B dissection. J Vasc Surg. 2008;48:465-8.

23. Katayama K, Uchida N, Takahashi S, Sueda T. Scheduled re-entry coil embolization before entry coverage of thoracic endovascular stent grafting for aneurysmal chronic type B aortic dissection. Interact Cardiovasc Thorac Surg. 2012;15:800-1.

24. Khaja MS, Park AW, Swee W, Evans AJ, Fritz Angle J, Turba UC, et al. Treatment of type II endoleak using Onyx with long-term imaging follow-up. Cardiovasc Intervent Radiol. 2014;37:613-22.

25. Chalouhi N, Starke RM, Tjoumakaris SI, Jabbour PM, Gonzalez LF, Hasan D, et al. Carotid and vertebral artery sacrifice with a combination of Onyx and coils: technical note and case series. Neuroradiology. 2013;55:993-8.

26. Gao X, Liang G, Li Z, Wang X, Yu C, Cao P, et al. Transarterial coil-augmented Onyx embolization for brain arteriovenous malformation: technique and experience in 22 consecutive patients. Interv Neuroradiol. 2014;20:83-90.

27. Cekirge HS, Saatci I, Geyik S, Yavuz K, Oztürk H, Pamuk G. Intrasaccular combination of metallic coils and onyx liquid embolic agent for the endovascular treatment of cerebral aneurysms. J Neurosurg. 2006;105:706-12.

28. Canaud L, Ozdemir BA, Patterson BO, Holt PJ, Loftus IM, Thompson MM Retrograde aortic dissection after thoracic endovascular aortic repair. Ann Surg. 2014;260:389-95.

29. Idrees J, Arafat A, Johnston DR, Svensson LG, Roselli EE. Repair of retrograde ascending dissection after descending stent grafting. J Thorac Cardiovasc Surg. 2014;147:151-4. 
30. Kpodonu J, Preventza O, Ramaiah VG, Shennib H, Wheatley GH III, RodriquezLopez J, et al. Retrograde type A dissection after endovascular stenting of the descending thoracic aorta: is the risk real? Eur J Cardiothorac Surg. 2008;33: 1014-8.

31. Ziza V, Canaud L, Molinari N, Branchereau P, Marty-Ané C, Alric P. Thoracic endovascular aortic repair: a single center's 15-year experience. J Thorac Cardiovasc Surg. 2016;151:1595-603.e7.

32. Eggebrecht H, Thompson M, Rousseau H, Czerny M, Lönn L, Mehta RH, et al. Retrograde ascending aortic dissection during or after thoracic aortic stent graft placement: insight from the European Registry on Endovascular Aortic Repair Complications. Circulation. 2009;120(11 Suppl):S276-81.

33. Mangialardi N, Serrao E, Kasemi H, Alberti V, Fazzini S, Ronchey S. Chimney technique for aortic arch pathologies: an 11-year single-center experience. J Endovasc Ther. 2014;21:312-23.

Key Words: aortic dissection, coil, onyx glue, retrograde, TEVAR

Readers who found these articles interesting may also like to read the following papers found in recent and future issues of our sister publications, Seminars in Thoracic and Cardiovascular Surgery and Operative Techniques in Thoracic and Cardiovascular Surgery!

\section{Acquired: Aortic Disease}

Original Submission: Outcomes of Open Surgical Repair for Type B Dissecting Aortic Aneurysm with Alternative Methods in the Endovascular Stent Era. Mitsumasa Hata. Semin Thorac Cardiovasc Surg 2015; Summer; 27(2):106-112.

Editorial Commentary: It is Difficult to Compare Apples and Oranges: Acute and Chronic Type B Aortic Dissections, Complicated and Uncomplicated, are Different and Should be Treated as Such. Ourania Preventza. Semin Thorac Cardiovasc Surg 2015; Summer; 27(2):113-114.

News and Views: Treatment of Thoracic Aortic Aneurysm: Role of Earlier Intervention. Bulat A. Ziganshin. Semin Thorac Cardiovasc Surg 2015; Summer; 27(2);135-143.

Aortic root enlargement during aortic valve replacement: Nicks and Manouguian techniques. Kendra J Grub. Oper Tech Thorac Cardiovasc Surg 2015; Autumn; 20(3):206-218.

The Konno-Rastan procedure for anterior aortic annular enlargement. Mark E. Roeser. Oper Tech Thorac Cardiovasc Surg 2015: Autumn; 20(3):219-233. 